Pacific Journal of Mathematics

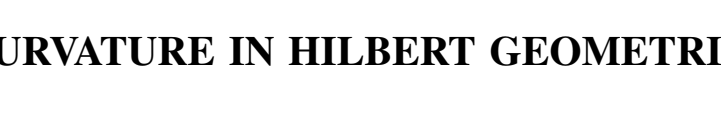




\section{CURVATURE IN HILBERT GEOMETRIES}

\section{Paul Kelly and ERnst Straus}

For every pair of points, $p$ and $q$, interior to a simple, closed, convex curve $C$ in the Euclidean plane, the line $\xi=p \times q$ cuts $C$ in a pair of points $u$ and $v$. If $C$ has at most one segment then the Hilbert distance from $p$ to $q$, defined by

$$
h(p, q)=\left|\log \left(\begin{array}{cc}
u p & v q \\
u q & v p
\end{array}\right)\right|,
$$

is a proper metric (where $u p$ denotes the Euclidean distance from $u$ to $p$ ), and is invariant under projective transformations. The geometry induced on the interior of $C$ is a Hilbert geometry, and the Hilbert lines are carried by Euclidean lines [2].

We shall be concerned here with curvature at a point defined in a qualitative rather than a quantitative sense (cf. [1, p 237]).

DEFINITION 1. The curvature at $p$ is positive or negative if there exists a neighborhood $U$ of $p$ such that for every $x, y$ in $U$ we have

$$
2 h(\bar{x}, \bar{y}) \geqq h(x, y) \text {, }
$$

respectively

$$
2 h(\bar{x}, \bar{y}) \leqq h(x, y),
$$

where $\bar{x}, \bar{y}$ are the Hilbert midpoints respectively of the segments from $p$ to $x$ and $p$ to $y$. If there is neither positive nor negative curvature at a point then the curvature is indeterminate at that point. This qualitative curvature is clearly a projective invariant.

In order to state our result we need one more concept.

Definition 2. A point $p$ is a projective center of $C$ if there exists a projective transformation, $\pi$, of the plane so that $\pi p$ is the affine center of $\pi C$.

A projective center is characterized by the following. Let $\xi$ be a line through $p$, and let $\xi n C=\{u, v\}$, and let $p_{\xi}^{\prime}$ be the harmonic conjugate of $p$ with respect to $u$ and $v$. Finally, let $L_{p}$ be the locus of all $p_{\xi}^{\prime}$. Then $\mathrm{p}$ is a projective center if and only if $L_{p}$ is a straight line.

Conic sections are characterized by the fact that every point in their interior is a projective center [3]. We can now state our main result, which solves a problem of $\mathrm{H}$. Busemann [1, Problem 34, p. 406].

THEOREM. If $p$ is a point of determinate curvature then it is 
a projective center of $C$. In particular, if the curvature is determinate everywhere then $C$ is an ellipse and the Hilbert geometry is hyperbolic.

We first establish some lemmas.

Lemma 1. For any point $p$, interior to $C$, there exists a line $\eta$ (possibly the line at infinity) which intersects $L_{p}$ in at least two points and does not intersect $C$.

Proof. There is at least one chord of $C$ which is bisected by $p$. If $\xi_{1}$ is the line of such a chord then $\xi_{1}$ intersects $L_{p}$ at $q_{1}$ on the line at infinity. If $L_{p}$ has a second point at infinity then the line at infinity satisfies the lemma. If $L_{p}$ has only one point at infinity then $L_{p}$ is a connected curve. It cannot lie within the strip formed by the two supporting lines of $C$ which are parallel to $\xi_{1}$ for then it would intersect $C$. There is therefore a point $q_{2}$ of $L_{p}$ outside this strip and the line $\eta=q_{1} \times q_{2}$ satisfies the lemma.

CoRollary. For every $p$ in the interior of $C$ there exists a projective transformation, $\pi$, so that $\pi C$ is a closed, convex curve, and so that $\pi p$ is the midpoint of two mutually perpendicular chords of $\pi C$ whose endpoints are points of differentiability of $\pi C$.

Proof. Since all but a denumerable set of points of $C$ are points of differentiability, we may choose the line $\eta$ of Lemma 1 so that $\eta \cap L_{p}$ contains $p_{\xi_{1}}^{\prime}$ and $p_{\xi_{2}}^{\prime}$ and so that $C$ is differentiable at its points of intersection with $\xi_{1}$ and $\xi_{2}$. Now let $\pi_{1}$ be a projective transformation which maps $\eta$ into the line at infinity, and let $\pi_{2}$ be an affine transformation which maps $\pi_{1} \xi_{1}$ and $\pi_{1} \xi_{2}$ into perpendicular lines. Then $\pi=\pi_{2} \pi_{1}$ has the required properties.

Lemma 2. If a chord of $C$, of (Euclidean) length $2 k$, has $p$ for its midpoint and if $q$ is a neighboring point on the chord at (Euclidean) distance ds from $p$, then $d S=(2 / k) d s+O\left(d s^{3}\right)$, where $d S=h(p, q)$.

Proof. If the endpoints of the chord are $u$ and $v$, and the order of the points on the chords is $u, p, q, v$, then, by definition,

$$
\begin{aligned}
d S= & \log \left(\frac{u p+p q}{u p}\right)\left(\frac{v p}{v p-p q}\right)=\log \left(\frac{k+d s}{k}\right)\left(\frac{k}{k-d s}\right) \\
= & \log \left(1+\frac{d s}{k}\right)-\log \left(1-\frac{d s}{k}\right) \\
= & {\left[\frac{d s}{k}-\frac{1}{2}\left(\frac{d s}{k}\right)^{2}+\frac{1}{3}\left(\frac{d s}{k}\right)^{3}-\cdots\right] } \\
& \quad-\left[-\frac{d s}{k}-\frac{1}{2}\left(\frac{d s}{k}\right)^{2}-\frac{1}{3}\left(\frac{d s}{k}\right)^{3}-\cdots\right]
\end{aligned}
$$




$$
=\frac{2}{k} d s+O\left(d s^{3}\right)
$$

Lemma 3. Let $(r, \theta)$ be polar coordinates whose pole $p$ is an interior point of $C$ at which the curvature is determinate. If $C$ is differentiable at the ends of two perpendicular chords which bisect each other at $p$, then $C$ satisfies the "one-sided" differential relations

$$
\begin{aligned}
& \left.\frac{d}{d \theta}\left(\begin{array}{c}
\csc 2 \theta \\
r^{2}
\end{array}\right)\right|_{\theta_{0}^{+}}=\left.\frac{d}{d \theta}\left(\frac{\csc 2 \theta}{r^{2}}\right)\right|_{\left(\theta_{0}+\pi\right)^{+}} \\
& \left.d\left(\frac{\csc 2 \theta}{r^{2}}\right)\right|_{\theta_{0}^{-}}=\left.\frac{d}{d \theta}\left(\frac{\csc 2 \theta}{r^{2}}\right)\right|_{\left(\theta_{0}+\pi\right)^{-}}
\end{aligned}
$$

for all $\theta_{0}$.

Proof. We first introduce Cartesian coordinates, with origin $p$, so that the $y$-axis intersects $C$ at points of second order differentiability, and so that the axes do not coincide with the two given chords bisected by $p$. The curve $C$ is then given by an "upper" arc $y=y_{1}(x)$ and a "lower" arc $y=-y_{2}(x)$. Let the bisected chords lie on the lines $\xi_{1}: y=a x$ and $\xi_{2}: y=(1 / a) x$ respectively. Let $b_{1}=(d x, a d x)$ and $c_{1}=(2 d x$, $2 a d x)$ on $\xi_{1}$, and $b_{2}=(d x,-(1 / a) d x)$ and $c_{2}=(2 d x,-(2 / a) d x)$ on $\xi_{2}$, where $d x$ is positive and chosen so that $b_{1}, b_{2}, c_{1}$, and $c_{2}$ lie inside $C$. Assume that $p$ is a point of negative curvature. Then.

$$
2 h\left(m_{1}, m_{2}\right) \leqq h\left(c_{1}, c_{2}\right) .
$$

where $m_{i}$ is the Hilbert midpoint of the segment from $p$ to $c_{i}$.

To show that $h\left(m_{i}, b_{i}\right)=O\left(d x^{3}\right)$, we define $d S_{1}=h\left(p, c_{1}\right)$ and $d s_{1}=p c_{1}$. With $2 k$ representing the Euclidean length of the chord on $\xi_{1}$, it follows from Lemma 2 that $d S_{1}=(2 / k) d s_{1}+O\left(d s_{1}^{3}\right)$, and hence that

$$
h\left(p, m_{1}\right)=\frac{1}{2} d S_{1}=\frac{1}{k} d s_{1}+O\left(d s_{1}^{3}\right) .
$$

Also, from Lemma 2 and the relation $d s_{1}=2 p b_{1}$, it follows that

$$
h\left(p, b_{1}\right)=\frac{2}{k} p b_{1}+O\left[\left(p b_{1}\right)^{3}\right]=\frac{1}{k}\left(d s_{1}\right)+O\left(d s_{1}^{3}\right) .
$$

Since $h\left(m_{1}, b_{1}\right)=\left|h\left(p, m_{1}\right)-h\left(p, b_{1}\right)\right|$, equations (3) and (4) imply that $h\left(m_{1}, b_{1}\right)$ $=O\left(d s_{1}^{3}\right)$. But $d s_{1}=d x\left(1+a^{2}\right)^{1 / 2}=O(d x)$, hence $h\left(m_{1}, b_{1}\right)=O\left(d x^{3}\right)$. Similarly, $h\left(m_{2}, b_{2}\right)=O\left(d x^{3}\right)$, and therefore

$$
h\left(m_{1}, b_{1}\right)+h\left(m_{2}, b_{2}\right)=O\left(d x^{3}\right) .
$$

From the triangle inequality, 
(6)

$$
h\left(m_{1}, m_{2}\right) \geqq h\left(b_{1} b_{2}\right)-h\left(m_{1}, b_{1}\right)-h\left(m_{2}, b_{2}\right) .
$$

This, together with (5), yields

$$
h\left(m_{1}, m_{2}\right) \geqq h\left(b_{1}, b_{2}\right)-O\left(d x^{3}\right),
$$

and from (1) and (7) we obtain

$$
2 h\left(b_{1}, b_{2}\right)<h\left(c_{1}, c_{2}\right)+O\left(d x^{3}\right) .
$$

We now wish to calculate the distances in (8). First, we have

$$
\begin{aligned}
h\left(b_{1}, b_{2}\right)= & h\left[(d x, a d x),\left(d x,-\frac{1}{a} d x\right)\right] \\
= & \log \left[\begin{array}{ll}
y_{1}(d x)+\frac{1}{a} d x & y_{2}(d x)+a d x \\
y_{1}(d x)-a d x & y_{2}(d x)-\frac{1}{a} d x
\end{array}\right] \\
= & \log \left[\begin{array}{l}
\left.1+\frac{d x}{a y_{1}(d x)}\right]+\log \left[1+\frac{a d x}{y_{2}(d x)}\right] \\
\quad-\log \left[1-\frac{a d x}{y_{1}(d x)}\right]-\log \left[1-\frac{d x}{a y_{2}(d x)}\right]
\end{array}\right.
\end{aligned}
$$

Using the Maclaurin expansion of the logarithms, and collocting first and second degree terms, we obtain

$$
\begin{aligned}
h\left(b_{1}, b_{2}\right)=d x & \left(a+\frac{1}{a}\right)\left[\frac{1}{y_{1}(d x)}+\frac{1}{y_{2}(d x)}\right] \\
& +\frac{d x^{2}}{2}\left(a^{2}-\frac{1}{a^{2}}\right)\left[\frac{1}{y_{1}^{2}(d x)}-\frac{1}{y_{2}^{2}(d x)}\right]+O\left(d x^{3}\right) .
\end{aligned}
$$

Because both of the functions $y_{1}(x)$ and $y_{2}(x)$ are convex and have second derivatives at $x=0$, they can be represented in the form

$$
y_{i}(d x)=y_{i}(0)+y_{i}^{\prime}(0) d x+O\left(d x^{2}\right), \quad i=1,2,
$$

and hence

$$
\begin{aligned}
& \frac{1}{y_{i}(d x)}=-\frac{1}{y_{i}(0)}-\frac{y_{i}^{\prime}(0)}{y_{i}^{2}(0)} d x+O\left(d x^{2}\right) \\
& \frac{1}{y_{i}^{2}(d x)}=\frac{1}{y_{i}^{2}(O)}+O(d x) .
\end{aligned}
$$

The substitution of (12) in (10) gives

$$
\begin{aligned}
h\left(b_{1}, b_{2}\right) & =d x\left(a+\frac{1}{a}\right)\left[\frac{1}{y_{1}}-\frac{y_{1}^{\prime} d x}{y_{1}^{2}}+\frac{1}{y_{2}^{2}}-\frac{y_{1}^{\prime} d x}{y_{2}^{2}}\right] \\
& +\frac{d x^{2}}{2}\left(a^{2}-\frac{1}{a^{2}}\right)\left(\frac{1}{y_{1}^{2}}-\frac{1}{y_{2}^{2}}\right)+O\left(d x^{3}\right),
\end{aligned}
$$


where $y_{i}=y_{i}(0)$. Hence

$$
\begin{aligned}
2 h\left(b_{1}, b_{2}\right) & =2 d x\left(a+\frac{1}{a}\right)\left[\frac{1}{y_{1}}+\frac{1}{y_{2}}-\frac{y_{1}^{\prime} d x}{y_{1}^{2}}-\frac{y_{2}^{\prime} d x}{y_{2}^{2}}\right. \\
& \left.+\frac{d x}{2}\left(a-\frac{1}{a}\right)\left(\frac{1}{y_{1}^{2}}-\frac{1}{y_{2}^{2}}\right)\right]+O\left(d x^{3}\right) .
\end{aligned}
$$

By the substitution of $2 d x$ for $d x$ we obtain

$$
\begin{aligned}
h\left(c_{1}, c_{2}\right) & =2 d x\left(a+\frac{1}{a}\right)\left[\frac{1}{y_{1}}+\frac{1}{y_{2}}-\frac{2 y_{1}^{\prime} d x}{y_{1}^{2}}-\frac{2 y_{2}^{\prime} d x}{y_{2}^{2}}\right. \\
& \left.+d x\left(a-\frac{1}{a}\right)\left(\begin{array}{c}
1 \\
y_{1}^{2}
\end{array}-\frac{1}{y_{2}^{2}}\right)\right]+O\left(d x^{3}\right) .
\end{aligned}
$$

Substituting this and (14) in (8) we have

$$
\begin{aligned}
2 d x\left(a+\frac{1}{a}\right)\left[\begin{array}{c}
1 \\
y_{1}
\end{array}\right. & \frac{1}{y_{2}}-\frac{y_{1}^{\prime} d x}{y_{1}^{2}}-y_{2}^{\prime} d x \\
& \left.+\frac{d x}{2}\left(a-\frac{1}{a}\right)\left(\frac{1}{y_{1}^{2}}-\frac{1}{y_{2}^{2}}\right)\right] \\
<2 d x\left(a+\frac{1}{a}\right)\left[\begin{array}{c}
1 \\
y_{1}
\end{array}\right. & \frac{1}{y_{2}}-\frac{2 y_{1}^{\prime} d x}{y_{1}^{2}}-\frac{2 y_{2}^{\prime} d x}{y_{2}^{2}} \\
& \left.+d x\left(a-\frac{1}{a}\right)\left(\frac{1}{y_{1}^{2}}-\frac{1}{y_{2}^{2}}\right)\right]+O\left(d x^{3}\right) .
\end{aligned}
$$

By dividing both sides of this inequality by $2 d x(a+1 / a)$, and then rearranging the terms, we obtain

$$
d x\left(\frac{y_{1}^{\prime}}{y_{1}}+\frac{y_{2}^{\prime}}{y_{2}}\right)-\frac{d x}{2}\left(a-\frac{1}{a}\right)\left(\frac{1}{y_{1}^{2}}-\frac{1}{y_{2}^{2}}\right)<O\left(d x^{2}\right) .
$$

Division of both sides of (17) by $d x$ yields a new inequality whose right side is $O(d x)$ but whose left side is independent of $d x$. From this it follows that

$$
\frac{y_{1}^{\prime}}{y_{1}}+\frac{y_{2}^{\prime}}{y_{2}}-\frac{1}{2}\left(a-\frac{1}{a}\right)\left(\frac{1}{y_{1}^{2}}-\frac{1}{y_{2}^{2}}\right) \leqq 0
$$

Consider now a reflection in the $y$-axis taking $C$ into a curve $\bar{C}$ which is divided by the $x$-axis into an "upper" $\operatorname{arc} z=z_{1}(x)$ and a "lower" arc $z=-z_{2}(x)$. With the lines $z=(1 / a)$ and $z=-a x$ playing the roles of $\xi_{1}$ and $\xi_{2}$, and with $\bar{b}_{1}, \bar{c}_{1}, \bar{b}_{2}, \bar{c}_{2}$ defined respectively by $(d x,(1 / a) d x),(2 d x$, $(2 / a) d x),(d x,-a d x)$, and $(2 d x,-2 a d x)$, a repetition of the former argument leads to

$$
\frac{z_{1}^{\prime}}{z_{1}^{2}}+\frac{z_{2}^{\prime}}{z_{2}^{2}}-\frac{d x}{2}\left(\frac{1}{a}-a\right)\left(-\frac{1}{z_{1}^{2}}-\frac{1}{z_{2}^{2}}\right) \leqq 0 .
$$

Since $z_{i}=y_{i}$ and $z_{i}^{\prime}=-y_{i}^{\prime},(19)$ is also 


$$
-\frac{y_{1}^{\prime}}{y_{1}^{2}}-\frac{y_{2}^{\prime}}{y_{2}^{2}}+\frac{d x}{2}\left(a-\frac{1}{a}\right)\left(\frac{1}{y_{1}^{2}}-\frac{1}{y_{2}^{2}}\right) \leqq 0 \text {. }
$$

Combining the opposite inequalities (18) and (20), we obtain

$$
\frac{y_{1}^{\prime}}{y_{1}^{2}}+\frac{y_{2}^{\prime}}{y_{2}^{2}}-\frac{1}{2}\left(a-\frac{1}{a}\right)\left(\frac{1}{y_{1}^{2}}-\frac{1}{y_{2}^{2}}\right)=0 \text {. }
$$

Since (21) is an equality, it is clear that the same result would have been obtained if all preceding inequalities has been reversed. In other words (21) holds if $p$ is a point of determinate curvature.

To express (21) in polar coordinates, let the polar axis be $\xi_{1}$ and let $\theta_{0}$ designate the angle between the polar axis and the upper half-line of the $y$-axis. The angles of inclination to the $x$-axis of the tangent lines to $C$ at $\left(0, y_{1}\right)$ and $\left(0, y_{2}\right)$ are $\alpha_{1}$ and $\alpha_{2}$ respectively and the clockwise angles from the radius vectors to the tangent lines at these points are $\omega_{1}$ and $\omega_{2}$. From the standard relationships between polar and Cartesian coordinates, it follows that

$$
\begin{aligned}
& y_{1}^{\prime}(0)=\tan \alpha_{1}=-\cot \omega_{1}=\left[\begin{array}{ll}
1 & \frac{d r}{r} \\
d \theta
\end{array}\right] \theta_{0} \\
& y_{2}^{\prime}(0)=-\tan \alpha_{2}=\cot \omega_{2}=\left[\begin{array}{ll}
\frac{1}{r} & \frac{d r}{d \theta}
\end{array}\right] \theta_{0}+\pi
\end{aligned}
$$

Also, by definition, $a=\cot \theta_{0}$ so $\frac{1}{2}\left(a-\frac{1}{a}\right)=\cot 2 \theta_{0}$. Substituting this and (22) in (21) we obtain

$$
\left[\begin{array}{cc}
-\frac{d}{r^{3}} & \frac{d r}{d \theta}
\end{array}\right]_{\theta_{0}}+\left[\begin{array}{cc}
1 & d r \\
-r^{3} & d \theta
\end{array}\right]_{\theta_{0}+\pi}-\left(\cot 2 \theta_{0}\right)\left[\begin{array}{c}
1 \\
r^{2}\left(\theta_{0}\right)
\end{array}-\frac{1}{r^{2}\left(\theta_{0}+\pi\right)}\right]=0
$$

and hence

$$
\left[\frac{1}{r^{3}} \frac{d r}{d \theta}+\frac{1}{r^{2}} \cot 2 \theta\right]_{\theta_{0}}=\left[\frac{1}{r^{3}} \frac{d r}{d \theta}+\frac{1}{r^{2}} 2 \theta\right]_{\theta_{0}+\pi} .
$$

Multiplying both sides of (24) by $2 \csc 2 \theta_{0}=2 \csc 2\left(\theta_{0}+\pi\right)$ we have

$$
\left.\frac{d}{d \theta} \frac{(\csc 2 \theta)}{r^{2}}\right|_{\theta_{0}}=\left.\frac{d}{d \theta} \frac{(\csc 2 \theta)}{r^{2}}\right|_{\theta_{0}+\pi} .
$$

Since (25) involves only first derivatives, it holds for all $\theta_{0}$ for which $r$ is differentiable at both $\theta_{0}$ and $\theta_{0}+\pi$. Since the one-sided derivative exists everywhere, we get the desired relations in (1), for all $\theta_{0}$, from the semi-continuity of the one sided derivative.

Proof of the Theorem. According to the corollary of Lemma 1 there is always a projective transformation such that, after the transformation, 
$p$ satisfies the conditions of Lemma 3. From (1) we obtain

$$
\int_{\theta_{0}}^{\theta} d\left(\frac{\csc 2 \theta}{r^{2}}\right)=\int_{\theta_{0}+\pi}^{\theta+\pi} d\left(\frac{\csc 2 \theta}{r^{2}}\right),
$$

where the integrals are Stieltjes intergrals and the interval $\left(\theta_{0}, \theta\right)$ does not contain a multiple of $\pi / 2$. Hence

where

$$
\frac{1}{r^{2}(\theta)}=\frac{1}{r^{2}(\theta+\pi)}+k_{j} \sin 2 \theta, k_{j}=\text { constant }
$$

$$
(j-1) \frac{\pi}{2} \leqq \theta \leqq j \frac{\pi}{2},(j=1,2,3,4)
$$

Since $r$ is differentiable at the points for which $\theta=0, \pi / 2, \pi, 3 \pi / 2$, we obtain from (27), upon differentiation at these points, the relations $k_{1}=k_{2}=k_{3}=k_{4}$. On the other hand, if we replace $\theta$ by $\theta+\pi$ in (27) we obtain the relations $k_{1}=-k_{3}$, and $k_{2}=-k_{4}$. In other words, $k_{j}=0$ and $r(\theta)=r(\theta+\pi)$. Since this shows $p$ to be a metric center, it was initially a projective center.

The last statement in the theorem is well known (see [3] and e.g. $[2, \mathrm{p} .164])$.

If a Hilbert metric is defined in the interior of an $n$-dimensional, convex surface $S$, the definitions for curvature and projective centers are unchanged. The metric for the space induces, on any plane through an interior point $p$, a two-dimensional Hilbert geometry. If $p$ is a point of determinate curvature, it is a two-dimensional projective center for every plane through it. Since the $L_{p}$ locus for every plane section is a line, it is easily seen that the total $L_{p}$ locus must be a plane and hence that $p$ is a projective center of $S$. If curvature is determinate everywhere then $S$ is an ellipsoid and the geometry is hyperbolic.

It seems probable that a Hilbert geometry can contain no points of positive curvature.

\section{REFERENCES}

1. H. Busemann, The geometry of geodesics, Academic Press, N.Y. 1957.

2. , and P. Kelly, Projective geometry and projective metrics, Academic Press, N.Y. 1953.

3. T. Kajima, On a characteristic property of the conic and the quadric,, Sci. Rep., Tohoku Univ. 8, 1919

University Of California, Santa Barbara And los Angeles. 



\section{PACIFIC JOURNAL OF MATHEMATICS}

\section{EDITORS}

David Gilbarg

Stanford University

Stanford, California

R. A. Beaumont

University of Washington

Seattle 5 , Washington
A. L. Whiteman

University of Southern California Los Angeles 7, California

E. G. Straus

University of California

Los Angeles 24, California

\section{ASSOCIATE EDITORS}
E. F. BECKENBACH
C. E. BURGESS
M. HALL
E. HEWITT
A. HORN
V. GANAPATHY IYER
R. D. JAMES
M. S. KNEBELMAN

L. NACHBIN

I. NIVEN

T. G. OSTROM

H. L. ROYDEN
M. M. SCHIFFEI

G. SZEKERES

F. WOLF

K. YOSIDA

\section{SUPPORTING INSTITUTIONS}

UNIVERSITY OF BRITISH COLUMBIA

CALIFORNIA INSTITUTE OF TECHNOLOGY

UNIVERSITY OF CALIFORNIA

MONTANA STATE UNIVERSITY

UNIVERSITY OF NEVADA

OREGON STATE COLLEGE

UNIVERSITY OF OREGON

OSAKA UNIVERSITY

UNIVERSITY OF SOUTHERN CALIFORNIA
STANFORD UNIVERSITY

UNIVERSITY OF TOKYO

UNIVERSITY OF UTAH

WASHINGTON STATE COLLEGE

UNIVERSITY OF WASHINGTON

AMERICAN MATHEMATICAL SOCIETY

CALIFORNIA RESEARCH CORPORATION

HUGHES AIRCRAFT COMPANY

THE RAMO-WOOLDRIDGE CORPORATION

Printed in Japan by Kokusai Bunken Insatsusha

(International Academic Printing Co., Ltd.), Tokyo, Japan 


\section{Pacific Journal of Mathematics}

\section{Vol. 8, No. 1 \\ March, 1958}

Shimshon A. Amitsur, Commutative linear differential operators ......... 1

Masahiko Atsuji, Uniform continuity of continuous functions of metric

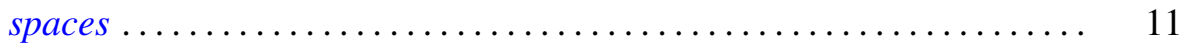

S. P. Avann, A numerical condition for modularity of a lattice . . . . . . . . . 17

Raymond G. D. Ayoub, A mean value theorem for quadratic fields........ 23

Errett Albert Bishop, Subalgebras of functions on a Riemann surface ..... . 29

Shaul Foguel, The relations between a spectral operator and its scalar

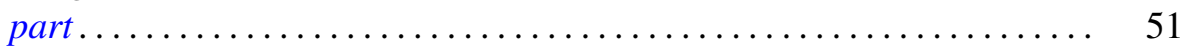

John Rolfe Isbell, Euclidean and weak uniformities ................. 67

Samuel Karlin and James L. McGregor, Many server queueing processes with Poisson input and exponential service times .............. 87

Paul Joseph Kelly and Ernst Gabor Straus, Curvature in Hilbert geometries....................................... 119

John W. Lamperti, Stationary measures for certain stochastic processes . . . 127

Richard Scott Pierce, Distributivity and the normal completion of Boolean

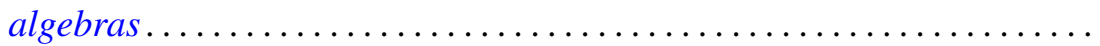

F. M. Ragab, Transcendental addition theorems for the hypergeometric

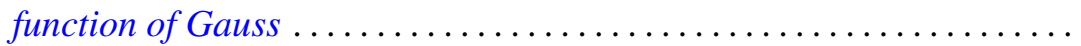

William T. Reid, Principal solutions of non-oscillatory self-adjoint linear differential systems ................................ 147

Maurice Sion, On general minimax theorems .................... 171

Chien Wenjen, On semi-normed ${ }^{*}$-algebras .................... 177 